\title{
A Dirichlet-Neumann cost functional approach for the Bernoulli problem
}

\author{
A. Ben Abda ${ }^{1}$, F. Bouchon ${ }^{2}$, G. H. Peichl ${ }^{3}$, M. Sayeh ${ }^{1}$ and \\ R. Touzani ${ }^{2}$ \\ ${ }^{1}$ LAMSIN, ENIT, El Manar University, Tunis, Tunisia \\ ${ }^{2}$ Clermont Université, Université Blaise Pascal, Laboratoire de Mathématiques, \\ BP10448, F-63000 Clermont-Ferrand, France and CNRS, UMR 6620, Laboratoire de \\ Mathématiques, F-63177 Aubière, France \\ ${ }^{3}$ University of Graz, Institute for Mathematics and Scientific Computing, \\ Heinrichstr. 36, 8010 Graz, Austria
}

\begin{abstract}
The Bernoulli problem is rephrased into a shape optimization problem. In particular, the cost function, which turns out to be a constitutive law gap functional, is borrowed to Inverse Problem formulations. The shape derivative of the cost functional is explicitly determined. The gradient information is combined with the level set method in a steepest descent algorithm to solve the shape optimization problem. The efficiency of this approach is illustrated by numerical results for both interior and exterior Bernoulli problems.
\end{abstract}

Keywords: Bernoulli problem, domain perturbation, free boundary, level set method, shape optimization, shape derivative.

\section{Introduction}

The Bernoulli problem arises in various physical situations such as electrochemical machining [21], potential flow in fluid mechanics [12] and optimal insulation [1]. In addition, the Bernoulli problem appears generally as a prototype of stationary free boundary problems. For more details on the physical background and an exhaustive bibliography we refer to $[11,13]$.

Given a bounded domain $A \subset \mathbb{R}^{2}$, with boundary $\Upsilon_{0}$ and $\lambda<0$, the exterior Bernoulli problem consists in finding a bounded domain $B \supset \bar{A}$ with boundary $\Gamma$ and a function $u$ defined on $\Omega=B \backslash \bar{A}$ such that:

$$
\begin{cases}\Delta u=0 & \text { in } \Omega, \\ u=1 & \text { on } \Upsilon_{0}, \\ u=0 & \text { on } \Gamma, \\ \frac{\partial u}{\partial \nu}=\lambda & \text { on } \Gamma\end{cases}
$$


where $\nu$ is the outward unit normal to $\Gamma$. For the interior Bernoulli problem, the unknown domain $B$ is in included in the given domain $A$ (see Section 4 for more details). Existence of solutions of the exterior problem can be obtained by various ways, for example by variational methods [3] or by the method of sub- and super-solutions [5]. In general, solutions of Bernoulli's free boundary problem are not unique. However, uniqueness for the exterior problem was proved in the context of convex domains (see [15]). Uniqueness for the exterior problem is also proved in [2] for a given star-shaped domain, together with results on star-shapeness and convexity of the solution domain for the $p$-Laplacian. For the interior problem, in the case where $A$ is a disk, one can show that no solution exists if $\lambda$ is close to 0 and that two solutions exist if $|\lambda|$ is sufficiently large. Thus, no result on existence or uniqueness of solution is available for the interior problem. For the qualitative theory and stability of solutions in dimension two, we refer to [13] and [15].

Several numerical methods $[7,13,14,16]$ have been developed to solve the twodimensional Bernoulli problem. In [14] this problem was transformed into the following shape optimization problem

$$
\min _{\Omega} J(\Omega)=\min _{\Omega} \frac{1}{2} \int_{\Gamma}\left(\frac{\partial u_{D}}{\partial \nu}-\lambda\right)^{2},
$$

where $u_{D}$ is the solution of the Dirichlet problem

$$
\begin{cases}\Delta u_{D}=0 & \text { in } \Omega, \\ u_{D}=1 & \text { on } \Upsilon_{0} \\ u_{D}=0 & \text { on } \Gamma .\end{cases}
$$

In [16] this problem is also transformed to a shape optimization problem exploiting the Neumann boundary condition,

$$
\min _{\Omega} J(\Omega)=\min _{\Omega} \frac{1}{2} \int_{\Gamma} u_{N}^{2},
$$

where $u_{N}$ is the solution of the Neumann problem

$$
\begin{cases}\Delta u_{N}=0 & \text { in } \Omega \\ u_{N}=1 & \text { on } \Upsilon_{0} \\ \frac{\partial u_{N}}{\partial \nu}=\lambda & \text { on } \Gamma\end{cases}
$$

As in $[14,16]$, we are interested in formulating the Bernoulli problem as a shape optimization problem. The originality of this approach relies on the use of an error functional that can be interpreted as a constitutive law error functional. This approach has been introduced in [22] in the context of a posteriori estimator in the finite element method. In this context, the minimization of the constitutive law error functional allows to detect the reliability of the mesh without knowing the exact solution. Within the 
inverse problem community this functional has been introduced in [20] in the context of parameter identification. It has also been used for Robin type boundary condition recovering [9] and in the context of geometrical flaws identification (see [8] and references therein). Here, the error functional depending on the domain $\Omega$ is defined by

$$
J(\Omega):=\frac{1}{2} \int_{\Omega}\left|\nabla u_{D}-\nabla u_{N}\right|^{2}
$$

Let us consider the subspace $V$ of $H^{1}(\Omega)$

$$
V=\left\{v \in H^{1}(\Omega) ; v=0 \text { on } \Upsilon_{0}\right\}
$$

and define the semi-norm on $H^{1}(\Omega)$

$$
|v|_{H^{1}(\Omega)}:=\left(\int_{\Omega}|\nabla v|^{2}\right)^{\frac{1}{2}} .
$$

We recall that $|\cdot|_{H^{1}(\Omega)}$ is a norm on $H_{0}^{1}(\Omega)$ and $V$, which thanks to the Poincaré inequality is equivalent to the norm of $H^{1}(\Omega)$.

If $(u, \Omega)$ is a solution of $(1)$ then $u_{D}=u_{N}=u$, therefore $J(\Omega)=0$. Conversely if $J(\Omega)=0$, since $u_{D}-u_{N} \in V, J(\Omega)=\frac{1}{2}\left|u_{D}-u_{N}\right|_{H^{1}(\Omega)}^{2}$ and $|\cdot|_{H^{1}(\Omega)}$ is a norm on $V$, then $u_{D}=u_{N}$ and $u=u_{D}=u_{N}$ is a solution of Problem (1). Therefore $(u, \Omega)$ is a solution of (1) if and only if $J(\Omega)=0$. Thus Problem (1) is equivalent to the shape optimization problem: find $(u, \Omega)$ such that

$$
J(\Omega)=\min _{\tilde{\Omega}} J(\tilde{\Omega})=0 .
$$

The main contribution of the present paper is to numerically solve the shape optimization problem (5).

We point out that the same cost functional $J$ was studied in [19] in 2009. We discovered that when we were preparing this paper. However, although we use the same cost functional, our contribution gives some worthfull results which hasn't been obtained in [19]. Firstly, in [19] the shape derivative has been determined with a shape calculus via boundary variations, developed in $[17,18]$ while we have determined it with an other way here, using the thechniques developped in [16] wich allow to compute the shape derivative for a perturbation $h \in C^{1, \alpha}$. Secondly we have treated also the interior case while the exterior one has been only treated in [19]. thirdly we have done more numerical tests espetially that of topology change and interior case.

The remainder of this paper is organized as follows: In Section 2 we prove existence of the shape derivative of the functional $J$, and give its analytical expression for the exterior Bernoulli problem together with the proof of this expression. In Section 3, we give a similar result for the interior Bernoulli problem without proof. In Section 4 
we describe how the level set method is used combined with the shape derivative to obtain numerical solutions for the Bernoulli problem and outline the algorithm to be implemented. In Section 5 some numerical results for the exterior and interior problems are given.

\section{Shape derivative of the functional $J$}

Let us consider a hold-all domain $U \supset \bar{\Omega}$ and introduce the mapping $F_{t}$ which defines a perturbation of the domain $\Omega$ of the form

$$
F_{t}=i d+t h
$$

where $i d$ is the identity operator, $h$ is a deformation field belonging to the space

$$
Q=\left\{h \in \mathcal{C}^{1,1}(\bar{U})^{2} ; h=0 \text { on } \Upsilon_{0}\right\},
$$

and $t$ is sufficiently small such that $F_{t}$ is a diffeomorphism from $\Omega$ onto its image. The perturbations of $\Omega$ and $\Gamma$ are respectively defined by

$$
\Omega_{t}:=F_{t}(\Omega), \quad \Gamma_{t}:=F_{t}(\Gamma) .
$$

Since $h$ vanishes on $\Upsilon_{0}$ for $h \in Q$, then $F_{t}\left(\Upsilon_{0}\right)=\Upsilon_{0}$. Thus, for all $t, \Upsilon_{0}$ is a part of the boundary of $\Omega_{t}$.

We consider the solutions $u_{D t}:=u_{D}\left(\Omega_{t}\right)$ and $u_{N t}:=u_{N}\left(\Omega_{t}\right)$ of (2) and (3) respectively formulated on the perturbed domain $\Omega_{t}$ instead of $\Omega$, i.e.

$$
\begin{aligned}
& \begin{cases}\Delta u_{D t}=0 & \text { in } \Omega_{t}, \\
u_{D t}=1 & \text { on } \Upsilon_{0}, \\
u_{D t}=0 & \text { on } \Gamma_{t},\end{cases} \\
& \begin{cases}\Delta u_{N t}=0 & \text { in } \Omega_{t}, \\
u_{N t}=1 & \text { on } \Upsilon_{0}, \\
\frac{\partial u_{N t}}{\partial \nu_{t}}=\lambda & \text { on } \Gamma_{t},\end{cases}
\end{aligned}
$$

where $\nu_{t}$ is the outward unit normal vector to $\Gamma_{t}$.

Definition 1. The Eulerian derivative of the functional $J$ at $\Omega$ in the direction of an element $h \in Q$ is defined by the quantity, when it exists:

$$
J^{\prime}(\Omega, h)=\lim _{t \rightarrow 0} \frac{J\left(\Omega_{t}\right)-J(\Omega)}{t} .
$$

The Eulerian derivative is called shape derivative if $J^{\prime}(\Omega, h)$ exists for all $h \in Q$ and the mapping $h \mapsto J^{\prime}(\Omega, h)$ is linear and continuous with respect to the topology of $\mathcal{C}^{1,1}(\bar{\Omega})^{2}$. 
Remark 1. If for $h \in Q$ the mapping $t \mapsto J\left(\Omega_{t}\right)$ is $\mathcal{C}^{1}$ in a neighborhood of 0 then the Eulerian derivative of $J$ in the direction of $h$ exists and we have

$$
J^{\prime}(\Omega, h)=\left.\frac{d J\left(\Omega_{t}\right)}{d t}\right|_{t=0}
$$

Let us now recall the Zolesio-Hadamard structure theorem (see [10]):

Theorem 1. There exists a function $G$ defined on $\Gamma$ such that

$$
J^{\prime}(\Omega, h)=\int_{\Gamma} G(h \cdot \nu) .
$$

We aim at proving existence of the shape derivative and deriving the analytic expression of the function $G$.

An advantage of the shape derivative structure (8) is that the gradient flow can be chosen obviously to minimize the functional $J$. More precisely

$$
h \in Q \quad \text { such that } h_{\mid \Gamma}=-G \nu,
$$

is a descent direction of the functional $J$. Thus, perturbing the domain in the direction defined by (9) decreases the value of $J$. In Section 5 we use this normal velocity and the level set method to numerically solve the shape optimization problem (5).

To prove existence and determine the shape derivative we first recall some transformation and differentiation formulas in the following two lemmas (see $[23,28]$ ). In the sequel if $\varphi$ is a function defined on the perturbed domain $\Omega_{t}$ or interface $\Gamma_{t}$, we

note $\varphi^{t}$ the function $\varphi \circ F_{t}$ which is defined on the reference domain $\Omega$ or interface $\Gamma$, especially we consider the functions:

$$
u_{N}^{t}=u_{N t} \circ F_{t}, \quad u_{D}^{t}=u_{D t} \circ F_{t} .
$$

\section{Lemma 1.}

i) If $\varphi \in L^{1}\left(\Omega_{t}\right)$, Then $\varphi^{t} \in L^{1}(\Omega)$ and we have

$$
\int_{\Omega_{t}} \varphi=\int_{\Omega} \delta_{t} \varphi^{t},
$$

where $\delta_{t}=\operatorname{det}\left(D F_{t}\right)=\operatorname{det}\left(I+t \nabla h^{\top}\right)\left(D F_{t}\right.$ is the Jacobian matrix of $\left.F_{t}\right)$.

ii) If $\varphi \in H^{1}\left(\Omega_{t}\right)$, then $\varphi^{t} \in H^{1}(\Omega)$ and we have

$$
(\nabla \varphi) \circ F_{t}=M_{t} \nabla \varphi^{t}
$$

with $M_{t}=D F_{t}^{-T}$. 
iii) If $\varphi \in L^{1}\left(\Gamma_{t}\right)$, then $\varphi^{t} \in L^{1}(\Gamma)$ and we have

$$
\int_{\Gamma_{t}} \varphi=\int_{\Gamma} \omega_{t} \varphi^{t}
$$

with $\omega_{t}=\delta_{t}\left|M_{t} \nu\right|$

Lemma 2. The mapings $t \mapsto \delta_{t}, t \mapsto M_{t}$ and $t \mapsto \omega_{t}$ with values in $\mathcal{C}(\Omega), \mathcal{C}(\Omega)^{2 \times 2}$ and $\mathcal{C}(\Gamma)$ respectively, are $\mathcal{C}^{1}$ in a neighborhood of 0 and we have

$$
\begin{aligned}
\left.\frac{d \delta_{t}}{d t}\right|_{t=0} & =\operatorname{div} h, \\
\left.\frac{d M_{t}}{d t}\right|_{t=0} & =-\nabla h, \\
\left.\frac{d \omega_{t}}{d t}\right|_{t=0} & =\operatorname{div}_{\Gamma} h,
\end{aligned}
$$

where $\operatorname{div}_{\Gamma} h:=\operatorname{div} h_{\mid \Gamma}-\nabla h \nu \cdot \nu$ is the surface divergence of $h$ on $\Gamma$.

Using (10) and (11) and the fact that the mappings $t \mapsto \delta_{t}$ and $t \mapsto M_{t}$ are $\mathcal{C}^{1}$ in a neighborhood of 0 (see Lemma 2), we get

$$
J\left(\Omega_{t}\right)=\frac{1}{2} \int_{\Omega} \delta_{t}\left|M_{t}\left(\nabla u_{D}^{t}-\nabla u_{N}^{t}\right)\right|^{2} .
$$

To prove existence of the shape derivative, we first show that the mappings $t \mapsto u_{D}^{t}$ and $t \mapsto u_{N}^{t}$ are $\mathcal{C}^{1}$ in a neighborhood of 0 .

In the remaining of this section $h$ is a fixed element of the space $Q$. In Theorem 2 we prove that the mappings $t \mapsto u_{D}^{t}$ and $t \mapsto u_{N}^{t}$ are $\mathcal{C}^{1}$ in a neighborhood of 0 and we characterize their Eulerian derivatives at 0 in the direction $h$. The same type of result will then be proved for $t \mapsto J\left(\Omega_{t}\right)$ in Theorem 3 .

Theorem 2. The mappings $t \mapsto u_{D}^{t}$ and $t \mapsto u_{N}^{t}$ with values in $H^{1}(\Omega)$ are $\mathcal{C}^{1}$ in a neighborhood of 0 . Furthermore, their Eulerian derivatives at 0 , denoted by $\widetilde{u}_{D}$ and $\widetilde{u}_{N}$ respectively, satisfy the properties $\widetilde{u}_{D} \in H_{0}^{1}(\Omega), \widetilde{u}_{N} \in V$ and are solutions of the following respective variational problems:

$$
\begin{array}{ll}
\int_{\Omega} \nabla \widetilde{u}_{D} \cdot \nabla v=-\int_{\Omega} A \nabla u_{D} \cdot \nabla v & \forall v \in H_{0}^{1}(\Omega), \\
\int_{\Omega} \nabla \widetilde{u}_{N} \cdot \nabla v=-\int_{\Omega} A \nabla u_{N} \cdot \nabla v+\lambda \int_{\Gamma}\left(\operatorname{div}_{\Gamma} h\right) v & \forall v \in V,
\end{array}
$$

where $A=(\operatorname{div} h) I-\nabla h-(\nabla h)^{\top}$.

The proof of this theorem is based on the following two lemmas.

Lemma 3. The bilinear form $a_{t}$ defined on $V \times V$ by

$$
a_{t}(u, v)=\int_{\Omega} A_{t} \nabla u \cdot \nabla v \quad \forall u, v \in V,
$$

with $A_{t}=\delta_{t} M_{t}^{\top} M_{t}$, is continuous and coercive on $V \times V$ for $t$ sufficiently small. 
Proof. For $u, v \in V$ and $t$ sufficiently small, we have

$$
\left|a_{t}(u, v)\right| \leq\left\|A_{t}\right\|_{\infty}|u|_{H^{1}(\Omega)}|v|_{H^{1}(\Omega)},
$$

where $\|\cdot\|_{\infty}$ is the $L^{\infty}(\Omega)$-norm. Therefore $a_{t}$ is continuous.

Let us prove the coerciveness of $a_{t}$. We have

$$
a_{t}(u, u)=\int_{\Omega} A_{t} \nabla u \cdot \nabla u=\int_{\Omega}|\nabla u|^{2}+\int_{\Omega}\left(A_{t}-I\right) \nabla u \cdot \nabla u .
$$

Since the mapping $t \mapsto A_{t}$ is continuous at 0 , we have for $t$ sufficiently small

$$
1-\left\|A_{t}-I\right\|_{\infty}>0
$$

This implies

$$
a_{t}(u, u) \geq\left(1-\left\|A_{t}-I\right\|_{\infty}\right)|u|_{H^{1}(\Omega)}^{2} .
$$

then there is a constant $C(t)>0$ such that

$$
a_{t}(u, u) \geq C(t)|u|_{H^{1}(\Omega)}^{2} .
$$

Thanks to the previously mentioned equivalence of norms on $H_{0}^{1}(\Omega)$, we deduce the coerciveness of $a_{t}$ for $t$ small enough.

Lemma 4. The functions $u_{N}^{t}$ and $u_{D}^{t}$ are the unique solutions in $H^{1}(\Omega)$ of

$$
\begin{aligned}
& \begin{cases}u_{D}^{t}=1 \text { on } \Upsilon_{0}, \quad u_{D}^{t}=0 \text { on } \Gamma, & \\
\int_{\Omega} A_{t} \nabla u_{D}^{t} \cdot \nabla v=0 & \forall v \in H_{0}^{1}(\Omega),\end{cases} \\
& \begin{cases}u_{N}^{t}=1 \text { on } \Upsilon_{0}, & \forall v \in V, \\
\int_{\Omega} A_{t} \nabla u_{N}^{t} \cdot \nabla v=\lambda \int_{\Gamma} \omega_{t} v & \end{cases}
\end{aligned}
$$

where $A_{t}=\delta_{t} M_{t}^{\top} M_{t}$ and $\omega_{t}=\delta_{t}\left|M_{t} \nu\right|$.

Proof. The function $u_{N t} \in H^{1}\left(\Omega_{t}\right)$ is solution of the variational problem

$$
\left\{\begin{array}{l}
u_{N t}=1 \text { on } \Upsilon_{0}, \\
\int_{\Omega_{t}} \nabla u_{N t} \cdot \nabla \varphi=\lambda \int_{\Gamma_{t}} \varphi \quad \forall \varphi \in V_{t},
\end{array}\right.
$$

where

$$
V_{t}=\left\{\varphi \in H^{1}\left(\Omega_{t}\right) ; \varphi=0 \text { on } \Upsilon_{0}\right\} .
$$

Thus using (8) and (10) we have for all $\varphi \in V_{t}$

$$
\begin{aligned}
\int_{\Omega_{t}} \nabla u_{N t} \cdot \nabla \varphi & =\int_{\Omega} \delta_{t} M_{t} \nabla u_{N}^{t} \cdot M_{t} \nabla \varphi^{t} \\
& =\int_{\Omega} \delta_{t} M_{t}^{\top} M_{t} \nabla u_{N}^{t} \cdot \nabla \varphi^{t} \\
& =\int_{\Omega} A_{t} \nabla u_{N}^{t} \cdot \nabla \varphi^{t}
\end{aligned}
$$


We have also using (11),

$$
\int_{\Gamma_{t}} \varphi=\int_{\Gamma} \omega_{t} \varphi^{t}
$$

Therefore, taking $v=\varphi^{t}$ we obtain (16). We have $u_{N}^{t}-1 \in V$ and by (16)

$$
\int_{\Omega} A_{t} \nabla\left(u_{N}^{t}-1\right) \cdot \nabla v=\int_{\Gamma} \omega_{t} v \forall v \in V
$$

Since the bilinear form $a_{t}$ is continuous and coercive on $V \times V$ by Lemma 3 and the linear form $v \longmapsto \int_{\Gamma} \omega_{t} v$ is continuous on $V$, the Lax-Milgram theorem ensures that $u_{N}^{t}-1$ is the unique solution in $V$ of

$$
\int_{\Omega} A_{t} \nabla\left(u_{N}^{t}-1\right) \cdot \nabla v=\int_{\Gamma} \omega_{t} v \forall v \in V
$$

Hence $u_{N}^{t}$ is the unique solution of the variational problem $(16)$ in $H^{1}(\Omega)$.

Remark 2. Since the mappings $t \mapsto \delta_{t}$ and $t \mapsto M_{t}$ are $\mathcal{C}^{1}$ in a neighborhood of 0 , the mapping $t \mapsto A_{t}$, with values in $\mathcal{C}(\Omega)^{2 \times 2}$, is also $\mathcal{C}^{1}$ and we have

$$
\left.\frac{d A_{t}}{d t}\right|_{t=0}=A, \quad \text { with } A:=(\operatorname{div} h) I-\nabla h-(\nabla h)^{\top} .
$$

\section{Proof of Theorem 2}

Let us prove this result for the mapping $t \mapsto u_{N}^{t}$, the proof for $t \mapsto u_{D}^{t}$ being similar. We use for this the implicit function theorem. Using (16), we find that $u_{N}^{t}-u_{N}$ is the unique element of $V$ that satisfies

$$
\int_{\Omega} A_{t} \nabla\left(u_{N}^{t}-u_{N}\right) \cdot \nabla v=-\int_{\Omega} A_{t} \nabla u_{N} \cdot \nabla v+\lambda \int_{\Gamma} \omega_{t} v \quad \forall v \in V .
$$

We consider the function $\Phi: \mathcal{I} \times V \rightarrow V^{\prime}$ defined by

$$
<\Phi(t, w), v>:=\int_{\Omega} A_{t} \nabla\left(w+u_{N}\right) \cdot \nabla v-\lambda \int_{\Gamma} \omega_{t} v \quad \forall v, w \in V,
$$

where $V^{\prime}$ is the dual space of $V, \mathcal{I}$ is a neighborhood of 0 such that for each $t \in \mathcal{I}, F_{t}$ is a diffeomorphism of $\Omega$ onto $\Omega_{t}$ and the brackets $\langle\cdot, \cdot\rangle$ stand for the duality pairing of $V^{\prime} \times V$. Thus $u_{N}^{t}-u_{N}$ is the unique element in $V$ such that $\Phi\left(t, u_{N}^{t}-u_{N}\right)=0$. First, since the mappings $t \mapsto A_{t}$ and $t \mapsto \omega_{t}$ are $\mathcal{C}^{1}$ in a neighborhood of $0, \Phi$ is $\mathcal{C}^{1}$ and we have for all $v, w \in V$,

$$
<D_{w} \Phi(0,0) w, v>=\int_{\Omega} \nabla w \cdot \nabla v .
$$

Observing that the bilinear form in the right-hand side of (17) is continuous and coercive on $V$, we deduce from the Lax-Milgram theorem that $D_{w} \Phi(0,0)$ is an isomorphism from $V$ onto $V^{\prime}$. Using the implicit function theorem we conclude that the mapping 
$t \mapsto u_{N}^{t}-u_{N}$ is $\mathcal{C}^{1}$ in a neighborhood of 0 . Let $\widetilde{u}_{N} \in V$ be its derivative at 0 . Differentiating the identity $\Phi\left(t, u_{N}^{t}-u_{N}\right)=0$ with respect to $t$ we obtain at 0 :

$$
<D_{w} \Phi(0,0) \widetilde{u}_{N}, v>+<\frac{\partial \Phi}{\partial t}(0,0), v>=0 \quad \forall v \in V,
$$

which results in (14).

Theorem 3. The mapping $t \mapsto J(t)$ is $\mathcal{C}^{1}$ in a neighborhood of 0 and its derivative at 0 is given by

$$
J^{\prime}(\Omega, h)=\int_{\Gamma} G(h \cdot \nu)
$$

with

$$
G=\frac{1}{2}\left(\lambda^{2}-\left(\nabla u_{D} \cdot \nu\right)^{2}+2 \lambda \kappa u_{N}-\left(\nabla u_{N} \cdot \tau\right)^{2}\right),
$$

where $\tau$ is the unit tangent vector to $\Gamma$ and $\kappa$ is the curvature of $\Gamma$.

To prove this theorem we need the following lemma:

Lemma 5. The solutions $u_{D}$ and $u_{N}$ of problems (2) and (3), respectively satisfy the identities

$$
\begin{aligned}
& \int_{\Omega} A \nabla u_{D} \cdot \nabla u_{D}=-\int_{\Gamma}\left(\nabla u_{D} \cdot \nu\right)^{2} h \cdot \nu \\
& \int_{\Omega} A \nabla u_{N} \cdot \nabla u_{N}=\int_{\Gamma}\left|\nabla u_{N}\right|^{2} h \cdot \nu-2 \lambda \int_{\Gamma} h \cdot \nabla u_{N} .
\end{aligned}
$$

Proof. Using Green's formula and the fact that $h$ vanishes on $\Upsilon_{0}$, we have for all $u, v \in H^{1}(\Omega)$

$$
\begin{aligned}
-\int_{\Omega} \operatorname{div} h(\nabla u \cdot \nabla v) & =\int_{\Omega}(\operatorname{Hess}(u) \nabla v+\operatorname{Hess}(v) \nabla u) \cdot h-\int_{\Gamma}(\nabla u \cdot \nabla v) h \cdot \nu, \\
\int_{\Omega}(\nabla h)^{\top} \nabla v \cdot \nabla u & =-\int_{\Omega}(\Delta v) h \cdot \nabla u-\int_{\Omega} \operatorname{Hess}(u) \nabla v \cdot h+\int_{\Gamma}(\nabla v \cdot \nu)(h \cdot \nabla u), \\
\int_{\Omega} \nabla h \nabla v \cdot \nabla u & =\int_{\Omega}(\nabla h)^{\top} \nabla u \cdot \nabla v \\
& =-\int_{\Omega}(\Delta u) h \cdot \nabla v-\int_{\Omega} \operatorname{Hess}(v) \nabla u \cdot h+\int_{\Gamma}(\nabla u \cdot \nu)(h \cdot \nabla v),
\end{aligned}
$$

where Hess $(u)$ is the Hessian of $u$. From these identities, and using the fact that $A=(\operatorname{div} h) I-\nabla h-(\nabla h)^{\top}$, one obtains

$$
\begin{aligned}
-\int_{\Omega} A \nabla u \cdot \nabla v= & -\int_{\Omega} \Delta u(h \cdot \nabla v)-\int_{\Omega} \Delta v(h \cdot \nabla u)+\int_{\Gamma} \frac{\partial v}{\partial \nu}(h \cdot \nabla u) \\
& +\int_{\Gamma}(\nabla u \cdot \nu)(h \cdot \nabla v)-\int_{\Gamma}(\nabla u \cdot \nabla v) h \cdot \nu .
\end{aligned}
$$

Therefore, we get (19) and (20) for $u=v=u_{D}$ then for $u=v=u_{N}$ and by noting that

$$
\nabla u_{D}=\left(\frac{\partial u_{D}}{\partial \nu}\right) \nu, \quad \frac{\partial u_{N}}{\partial \nu}=\lambda, \quad \Delta u_{D}=\Delta u_{N}=0 .
$$




\section{Proof of Theorem 3}

We have

$$
J\left(\Omega_{t}\right)=\frac{1}{2} \int_{\Omega} \delta_{t}\left|M_{t}\left(\nabla u_{D}^{t}-\nabla u_{N}^{t}\right)\right|^{2}=\frac{1}{2} \int_{\Omega} A_{t}\left(\nabla u_{D}^{t}-\nabla u_{N}^{t}\right) \cdot\left(\nabla u_{D}^{t}-\nabla u_{N}^{t}\right) .
$$

Since the mappings $t \mapsto A_{t}$ and by Theorem $2 t \mapsto u_{N}^{t}$ and $t \mapsto u_{D}^{t}$ with values on $H^{1}(\Omega)$ are $\mathcal{C}^{1}$ in a neighborhood of 0 then the mapping $t \mapsto J\left(\Omega_{t}\right)$ is $\mathcal{C}^{1}$ and we have

$$
J^{\prime}(\Omega, h)=\frac{1}{2} \int_{\Omega} A\left(\nabla u_{D}-\nabla u_{N}\right) \cdot\left(\nabla u_{D}-\nabla u_{N}\right)+\int_{\Omega}\left(\nabla u_{D}-\nabla u_{N}\right) \cdot\left(\nabla \widetilde{u}_{D}-\nabla \widetilde{u}_{N}\right),
$$

where $\widetilde{u}_{D}$ and $\widetilde{u}_{N}$ are the solutions of (13) and (14) respectively.

Let us express $\int_{\Omega}\left(\nabla u_{D}-\nabla u_{N}\right) \cdot\left(\nabla \widetilde{u}_{D}-\nabla \widetilde{u}_{N}\right)$ by $u_{D}$ and $u_{N}$ only. As $\widetilde{u}_{D} \in H_{0}^{1}(\Omega)$ we have from the variational problems verified by $u_{D}$ and $u_{N}$

$$
\int_{\Omega} \nabla u_{D} \cdot \nabla \widetilde{u}_{D}=0 \quad \text { and } \int_{\Omega} \nabla u_{N} \cdot \nabla \widetilde{u}_{D}=\lambda \int_{\Gamma} \widetilde{u}_{D}=0
$$

Since $u_{D}-u_{N} \in V$, we have using (14)

$$
\int_{\Omega}\left(\nabla u_{D}-\nabla u_{N}\right) \cdot \nabla \widetilde{u}_{N}=-\int_{\Omega} A \nabla u_{N} \cdot \nabla\left(u_{D}-u_{N}\right)+\lambda \int_{\Gamma} \operatorname{div}_{\Gamma} h\left(u_{D}-u_{N}\right) .
$$

Thus, since $u_{D}=0$ on $\Gamma$

$$
\int_{\Omega}\left(\nabla u_{D}-\nabla u_{N}\right) \cdot\left(\nabla \widetilde{u}_{D}-\nabla \widetilde{u}_{N}\right)=\int_{\Omega} A \nabla u_{N} \cdot \nabla\left(u_{D}-u_{N}\right)+\lambda \int_{\Gamma} \operatorname{div}_{\Gamma} h u_{N} .
$$

Thus

$$
J^{\prime}(\Omega, h)=\frac{1}{2} \int_{\Omega} A \nabla u_{D} \cdot \nabla u_{D}-\frac{1}{2} \int_{\Omega} A \nabla u_{N} \cdot \nabla u_{N}+\lambda \int_{\Gamma} \operatorname{div}_{\Gamma} h u_{N} .
$$

Therefore using Lemma 5 we have

$$
J^{\prime}(\Omega, h)=-\frac{1}{2} \int_{\Gamma}\left(\nabla u_{D} \cdot \nu\right)^{2} h \cdot \nu+\lambda \int_{\Gamma}\left(h \cdot \nabla u_{N}+\operatorname{div}_{\Gamma} h u_{N}\right)-\frac{1}{2} \int_{\Gamma}\left|\nabla u_{N}\right|^{2} h \cdot \nu .
$$

By the following formula, which is valid when $\Gamma$ is $\mathcal{C}^{2}$

$$
\int_{\Gamma}\left(\nabla b \cdot V+b \operatorname{div}_{\Gamma} V\right)=\int_{\Gamma}\left(\frac{\partial b}{\partial \nu}+b \operatorname{div}_{\Gamma} \nu\right) V \cdot \nu
$$

we obtain

$$
\int_{\Gamma} h \cdot \nabla u_{N}+\operatorname{div}_{\Gamma} h u_{N}=\int_{\Gamma}\left(\lambda+u_{N} \operatorname{div}_{\Gamma} \nu\right) h \cdot \nu .
$$

Recalling that $\left|\nabla u_{N}\right|^{2}=\lambda^{2}+\left(\nabla u_{N} \cdot \tau\right)^{2}$ and $\operatorname{div}_{\Gamma} \nu=\kappa$, we eventually get

$$
J^{\prime}(\Omega, h)=\frac{1}{2} \int_{\Gamma}\left(\lambda^{2}-\left(\nabla u_{D} \cdot \nu\right)^{2}+2 \lambda u_{N} \kappa-\left(\nabla u_{N} \cdot \tau\right)^{2}\right) h \cdot \nu .
$$




\section{The interior Bernoulli problem}

Given a bounded domain $A \subset \mathbb{R}^{2}$, with boundary $\Upsilon_{0}$ and $\lambda>0$. The interior Bernoulli problem consists in finding a bounded domain $B \subset \bar{A}$ with boundary $\Gamma$ and a function $u$ defined on $\Omega=A \backslash \bar{B}$ such that

$$
\begin{cases}\Delta u=0 & \text { in } \Omega, \\ u=0 & \text { on } \Upsilon_{0}, \\ u=1 & \text { on } \Gamma, \\ \frac{\partial u}{\partial \nu}=\lambda & \text { on } \Gamma,\end{cases}
$$

where $\nu$ is the interior unit normal to $\Gamma$.

As for the exterior problem we consider the functions $u_{D}$ and $u_{N}$ solutions of the following problems

$$
\begin{aligned}
& \begin{cases}\Delta u_{D}=0 & \text { in } \Omega, \\
u_{D}=0 & \text { on } \Upsilon_{0}, \\
u_{D}=1 & \text { on } \Gamma,\end{cases} \\
& \begin{cases}\Delta u_{N}=0 & \text { in } \Omega, \\
u_{N}=0 & \text { on } \Upsilon_{0}, \\
\frac{\partial u_{N}}{\partial \nu}=\lambda & \text { on } \Gamma,\end{cases}
\end{aligned}
$$

and the functional $J$ defined by

$$
J(\Omega)=\frac{1}{2} \int_{\Omega}\left|\nabla u_{D}-\nabla u_{N}\right|^{2} .
$$

The shape derivative of the functional $J$ is given in the following theorem, whose proof will be skipped, this one being similar to that of Theorem 3 .

Theorem 4. The mapping $t \mapsto J(t)$ is $\mathcal{C}^{1}$ in a neighborhood of 0 and its derivative at 0 is given by the quantity

$$
J^{\prime}(\Omega, h)=\int_{\Gamma} G(h \cdot \nu)
$$

with

$$
G=\frac{1}{2}\left(\lambda^{2}-\left(\nabla u_{D} \cdot \nu\right)^{2}+2 \lambda \kappa\left(u_{N}-1\right)-\left(\nabla u_{N} \cdot \tau\right)^{2}\right)
$$

\section{Numerical approximation}

The numerical solution of the Bernoulli problem is considered by adopting an iterative method which consists in decreasing the value of the cost functional $J$ at each iteration. 
Let $\Gamma^{k}:=\Gamma$ be the free boundary at the $k$-th iteration. We consider for $t \geq 0$ the deformation of $\Gamma$, as in Section 2

$$
\Gamma_{t}^{k}:=\Gamma_{t}=\{x+\operatorname{th}(x) ; x \in \Gamma\},
$$

where $h_{\mid \Gamma}=-G \nu$ is the descent direction of the functional $J$. The expression of $G$ is given in (18) for the exterior Bernoulli problem and (25) for the interior Bernoulli problem. Therefore we set

$$
\Gamma^{k+1}:=\Gamma_{T},
$$

for a given "final time" $T$ whose value will be made precise below. To numerically implement this iterative method, we use the level set method.

\subsection{Level set formulation}

Level set methods introduced by Osher and Sethian [25] are computational techniques for tracking moving interfaces under a variety of complex motions, which allow to handle topology changes in an automatic way. They have been used with considerable success in a wide collection of models, including fluid mechanics, crystal growth, combustion, and medical imaging. For several optimization problems, the level set method has been successfully applied to compute optimal geometries [6, 26]. A general overview of the theory, numerical approximation, and range of applications may be found in [27, 24]. It consists in representing the interface $\Gamma_{t}$ as the zero level set of a time-dependent, implicit and smooth function $\varphi(\cdot, t)$ defined on a neighborhood of $\Gamma$, i.e.

$$
\Gamma_{t}=\{x ; \varphi(x, t)=0\},
$$

the function $\varphi(\cdot, t)$ being negative inside $\Gamma_{t}$ and positive outside. An advantage of the level set method is that geometric properties of the interface, such as the curvature $\kappa$ and the normal vector $\nu$, are naturally obtained from the level set function $\varphi$ as follows:

$$
\nu=\frac{\nabla \varphi}{|\nabla \varphi|}, \quad \kappa=\nabla \cdot \frac{\nabla \varphi}{|\nabla \varphi|} .
$$

Since $x+\operatorname{th}(x) \in \Gamma_{t}$ for $x \in \Gamma$, we have

$$
\varphi(x+t h(x), t)=0 \quad \forall(x, t) \in \Gamma \times[0, T] .
$$

Differentiating this identity with respect to $t$ and taking into account that

$$
h_{\mid \Gamma}=-G \nu=-G \frac{\nabla \varphi}{|\nabla \varphi|},
$$

we obtain the level set equation:

$$
\left\{\begin{array}{l}
\partial_{t} \varphi-G|\nabla \varphi|=0 \quad t \in(0, T], \\
\varphi(\cdot, 0)=\varphi_{0},
\end{array}\right.
$$

where $\varphi_{0}$ is a level set function corresponding to $\Gamma$. To solve this equation, the normal velocity $G$ needs to be extended in an appropriate way which we will explain below into a function $G_{e x t}$ in a neighborhood of $\Gamma$. 


\subsection{Approximation of the curvature and the speed function $G$}

For the numerical implementation we consider a square large enough containing $\Upsilon_{0}$ and define a uniform mesh of size $\delta x$. The level set functions are characterized by their nodal values on this mesh. A point $P$, with coordinate $x_{P}$, located on the interface between two nodes $\mathrm{M}$ and $\mathrm{N}$ of the mesh, with respective coordinates $x_{M}$ and $x_{N}$, is approximated using linear interpolation, i.e.

$$
x_{P}=x_{M}+\frac{\varphi\left(x_{M}\right)}{\varphi\left(x_{M}\right)-\varphi\left(x_{N}\right)}\left(x_{N}-x_{M}\right) .
$$

In a square cell intercepted by the interface, the interface is approximated by the segment linking the two points of the interface common with the boundary of this square cell (the segment $[P Q]$ in Fig. 1).

We use the $P_{1}$-finite element method to compute $u_{D}$ and $u_{N}$. For the approximation of the speed function $G$ on the interface $\Gamma$, the term $\left(\nabla u_{D} \cdot \nu\right)^{2}=\left|\nabla u_{D}\right|^{2}$ is directly deduced from the approximate $u_{D}$. The term $\nabla u_{N} \cdot \tau$ is approximated at $P$ by

$$
\left|\nabla u_{N} \cdot \tau\right| \approx \frac{\left|u_{N}\left(x_{P}\right)-u_{N}\left(x_{Q}\right)\right|}{\left|x_{P}-x_{Q}\right|}
$$

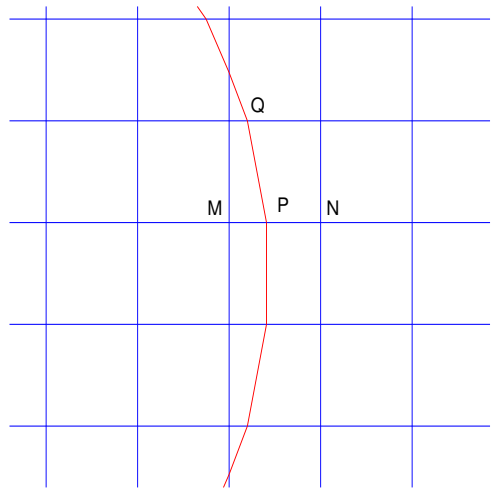

Figure 1. Finite difference approximation stencil.

For the approximation of the curvature at $P$ (Fig. 2), we take the points $\left(x_{i}, y_{i}\right)$ of the boundary located in the square $\left[x_{M}-k \delta x, x_{M}+k \delta x\right] \times\left[y_{M}-k \delta x, y_{M}+k \delta x\right]$ centered on $M$ (Fig. 2) and we construct a polynomial $p$ of degree 4 that fits the data $p\left(x_{i}\right)$ to $y_{i}$ (or $p\left(y_{i}\right)$ to $x_{i}$ ) in a least squares sense Fig. 2, and then we compute the local curvature using the classical formula

$$
\kappa=\frac{-p^{\prime \prime}}{\left(1+p^{\prime 2}\right)^{3 / 2}},
$$

where $k$ is an integer which is taken equal to $4,5, \ldots$, or 10 . 


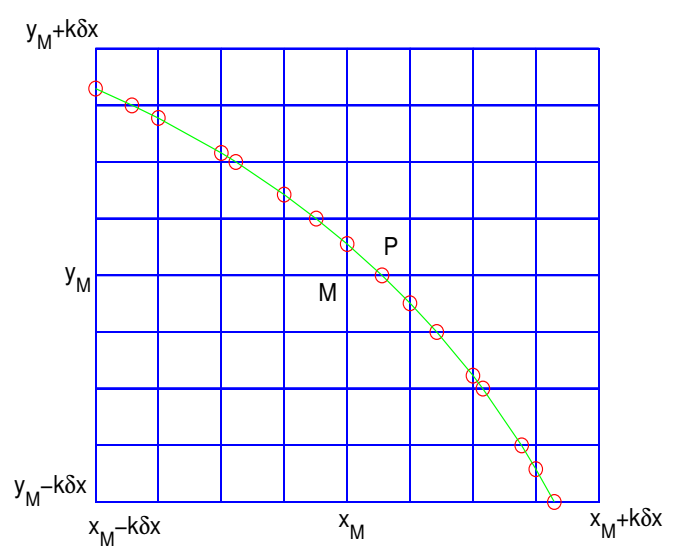

Figure 2. red ' $\circ$ ' points of the boundary located in the square $\left[x_{M}-k \delta x, x_{M}+k \delta x\right] \times$ $\left[y_{M}-k \delta x, y_{M}+k \delta x\right]$ centered on $M$ for $k=4$, green solid line fitting polynomial.

\subsection{Extension of the normal velocity}

The extension of the normal velocity $G$ into $G_{e x t}$ is designed so that the signed distance function is maintained as the interface moves. We use fast marching method [4] to simultaneously determine the signed distance function $\widehat{\varphi}_{0}$ to $\Gamma$ by solving:

$$
\left\{\begin{array}{l}
\left|\nabla \widehat{\varphi}_{0}\right|=1, \\
\widehat{\varphi}_{0}=0 \quad \text { on } \Gamma .
\end{array}\right.
$$

and extend the normal velocity $G$ into $G_{e x t}$ on a neighborhood of $\Gamma$ by solving:

$$
\left\{\begin{array}{l}
\nabla G_{e x t} \cdot \nabla \widehat{\varphi}_{0}=0, \\
G_{e x t}=G \quad \text { on } \Gamma .
\end{array}\right.
$$

\subsection{Updating the interface}

Once the normal velocity is extended and the signed distance function $\widehat{\varphi}_{0}$ is determined the level set equation (26) becomes

$$
\left\{\begin{array}{l}
\varphi_{t}-G_{e x t}|\nabla \varphi|=0 \\
\varphi(\cdot, 0)=\widehat{\varphi}_{0} \\
t \in[0, T]
\end{array}\right.
$$

As the extension of the normal velocity maintains the signed distance function we have $|\nabla \varphi| \approx 1$, thus we solve the level set equation by the following numerical scheme

$$
\varphi^{n+1}=\varphi^{n}+\delta t G_{e x t}
$$

where $\delta t$ is a small parameter that plays the role of a time step. 
Since the normal velocity depends on the curvature $\kappa$, the level set equation has a parabolic component. Thus numerical tests require that the time step satisfies

$$
d t=C(d x)^{2} .
$$

If necessary we reinitialize the level set equation after some time iteration using fast marching method $[27,4]$ by solving the Eikonal equation,

$$
\left\{\begin{array}{l}
|\nabla \varphi|=1, \\
\varphi=0 \quad \text { on } \Gamma .
\end{array}\right.
$$

We determine $T$ according to the following heuristic which is inspired by the ArmijoGoldstein line search strategy:

$$
J\left(\Gamma_{T}\right)=J\left(\Gamma_{0}\right)-\|G\|_{L^{2}\left(\Gamma_{0}\right)}^{2} T+o(T) \approx J\left(\Gamma_{0}\right)-\|G\|_{L^{2}\left(\Gamma_{0}\right)}^{2} T .
$$

Let us assume that $J\left(\Gamma_{T}\right)=\alpha J\left(\Gamma_{0}\right)$ for some $\alpha \in(0,1)$, then

$$
T=\frac{J(\Gamma)(1-\alpha)}{\|G\|_{L^{2}\left(\Gamma_{0}\right)}^{2}} .
$$

Finally we update the level set function by $\varphi^{n}$, where $n$ is the largest integer such that $n \delta t \leq T$.

\subsection{Stopping criterion}

To define a stopping criterion we consider the Hausdorff distance which is defined for two sets $A, B \subset \mathbb{R}^{2}$ by

$$
d_{H}(A, B)=\max \left(\sup _{a \in A} d(a, B), \sup _{b \in B} d(b, A)\right),
$$

where $d(a, B)=\inf _{b \in B}|a-b|$, the distances $d(a, b)$ being available via the level set functions. The stopping criterion of the algorithm is $d_{H}\left(\Gamma^{k}, \Gamma^{k-1}\right)<C \delta x^{2}$, where $C$ is a constant independent of $\delta x$.

\subsection{Outline of the algorithm}

Let us now summarize the proposed algorithm to be implemented for solving the Bernoulli problem. Assume that we know the interface $\Gamma^{k}:=\Gamma$ and a level set function $\varphi^{k}:=\varphi_{0}$ associated to $\Gamma^{k}:=\Gamma$.

(i) Compute the solutions $u_{D}$ and $u_{N}$ of the problems (2) and (3) respectively using $P_{1}$ finite element method.

(ii) Compute the speed function $G$ on $\Gamma$.

(iii) Simultaneously determine from $\varphi_{0}$ the signed distance function $\widehat{\varphi}_{0}$ to $\Gamma$ and extend $G$ to $G_{\text {ext }}$ in a neighborhood of $\Gamma$ using the fast marching method by solving (27) and (28).

(iv) Update the level set function $\varphi$ by solving the level set equation (29), via the numerical scheme (30). 


\section{Numerical tests}

We consider the square $[-1,1] \times[-1,1]$ as computational domain for all the following numerical tests except in the topology change test where the computational domain is $[-1.5,1.5] \times[-1.5,1.5]$.

\subsection{Exterior case}

In the first test for the exterior Bernoulli problem we take

$$
\Upsilon_{0}=\{x ;|x|=r\}, \quad \lambda=\frac{1}{R(\log r-\log R)},
$$

with $R>r>0$. In this case the only solution is the circle $C_{\text {ex }}$ centered at the origin with radius $R$ and the explicit expression $u_{e x}$ of $u$ is given by

$$
u_{e x}(x)=\frac{\log |x|-\log R}{\log r-\log R} .
$$

We have chosen $r=0.3$ and $R=0.5$ thus $\lambda=-3.9152$. We take as an initial guess the circle centered in the origin of radius 0.6. Table 1 shows the convergence test to the exact solution. Fig. 3 shows the convergence history by the decrease of the functional $J$ for both $32 \times 32,52 \times 52$ and $102 \times 102$ mesh grid.

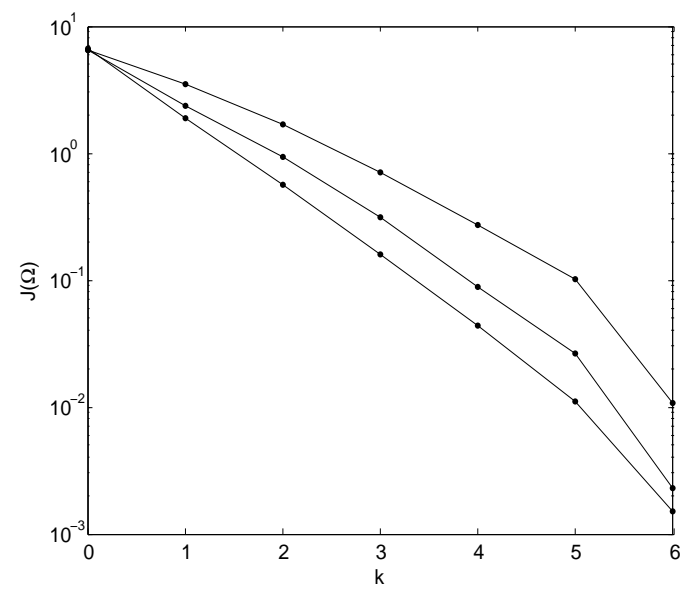

Figure 3. Convergence history for $32 \times 32,52 \times 52$ and $102 \times 102$ grids, top to bottom.

In the second test $\Upsilon_{0}$ is an L-shaped domain and $\lambda=-9$. We take as an initial guess the circle centered in the origin of radius 0.8 . We have obtained convergence after 10 iterations with $J\left(\Gamma^{10}\right)=0.0323$ for $152 \times 152$ mesh grid. Fig. 4 shows the evolution of the free boundary $\Gamma^{k}$ for $152 \times 152$ mesh grid, at different iterations.

The last test for the exterior Bernoulli problem is a topology change test. $\Upsilon_{0}$ is the union of two disjointed circles of radius 0.2 , one centered in $(-0.4,0)$ and the other in $(0.4,0)$. We take $\lambda=-1$, in this case the solution is connected, but we consider 
Table 1. Convergence test to the exact solution.

\begin{tabular}{lccll}
\hline Grid & Nb. Iter. & $d_{H}\left(\Gamma, C_{e x}\right)$ & Rate & $J(\Omega)$ \\
\hline $32 \times 32$ & 6 & 0.0108 & - & 0.0108 \\
$52 \times 52$ & 6 & 0.0050 & 1.54 & 0.0023 \\
$102 \times 102$ & 6 & 0.0032 & 0.65 & 0.0015 \\
$202 \times 202$ & 10 & 0.0024 & 0.41 & 0.0005 \\
$402 \times 402$ & 13 & 0.0014 & 0.78 & 0.0003 \\
\hline
\end{tabular}
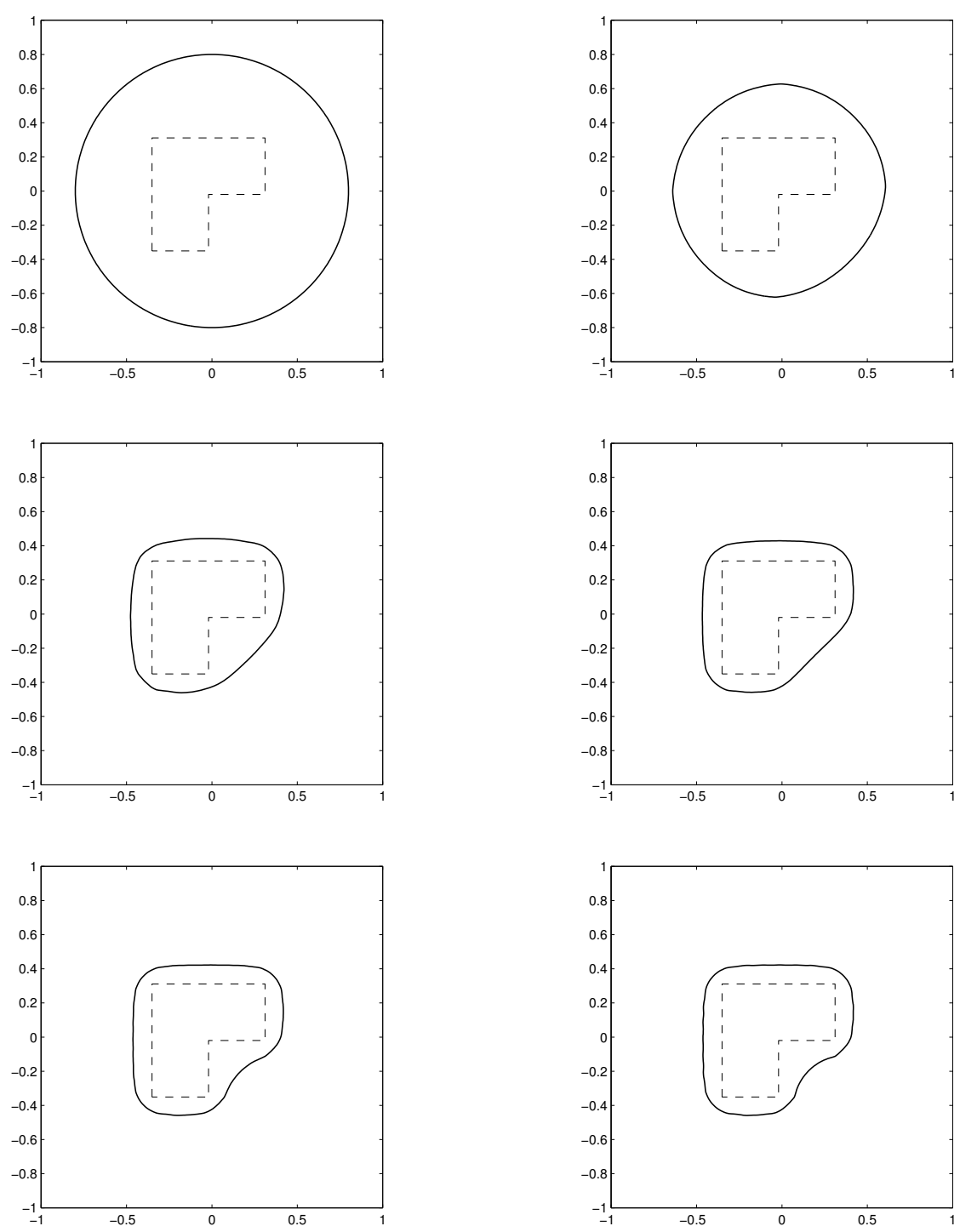

Figure 4. Position of $\Gamma^{k}$ for $k=0,1,4,5,8,10$ (left to right, top to bottom), $\Upsilon_{0}$ the dashed line and $\Gamma^{k}$ the solid line.

a disconnected initial guess: the union of the two disjointed circles of radius 0.3 , one centered in $(-0.4,0)$ and the other in $(0.4,0)$ as it is shown in Fig. 5. We have obtained convergence after 17 iterations with $J\left(\Gamma^{17}\right)=0.0005$ for $152 \times 152$ mesh grid. Fig. 5 
shows the evolution of the free boundary.
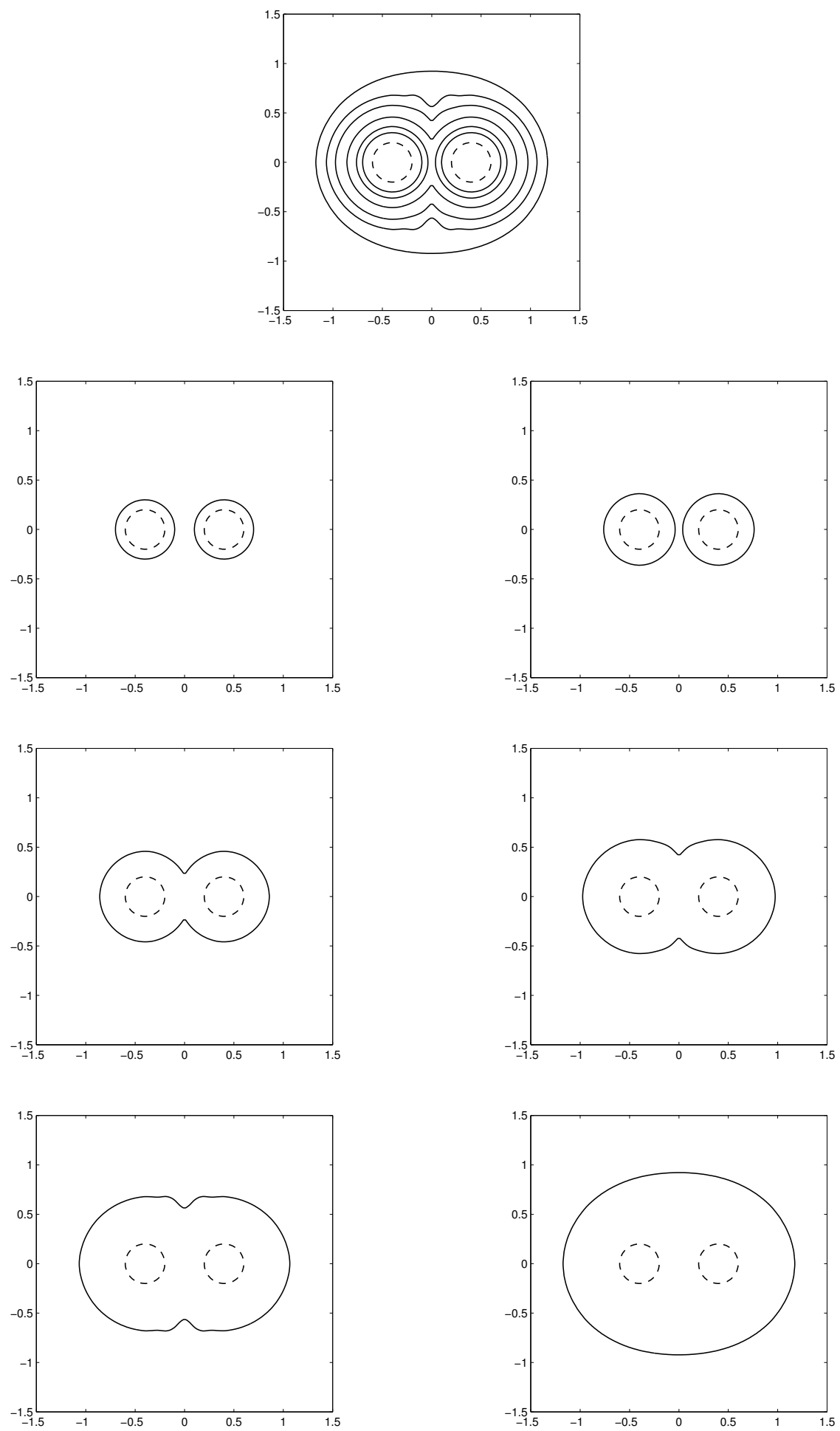

Figure 5. Topology change test: $\Upsilon_{0}$ the dashed line, evolution of the free boundary (solid line) $\Gamma^{k}$ for $k=0,1,2,3,4,17$ (left to right, top to bottom). 
Table 2. Convergence test to the elliptic solution.

\begin{tabular}{lccll}
\hline Grid & Nb. Iter. & $d_{H}\left(\Gamma, C_{e x}\right)$ & Rate & $J(\Omega)$ \\
\hline $52 \times 52$ & 8 & 0.0442 & - & 0.0023 \\
$102 \times 102$ & 24 & 0.0033 & 3.79 & 0.0015 \\
$202 \times 202$ & 33 & 0.0009 & 1.88 & 0.0005 \\
$402 \times 402$ & 54 & 0.0005 & 0.85 & 0.00004 \\
\hline
\end{tabular}

\subsection{Interior case}

In the first test for the interior Bernoulli problem we take $\Upsilon_{0}$ the circle

$$
\Upsilon_{0}=\{x ;|x|=R\}, \quad \lambda=\frac{1}{r(\log R-\log r)},
$$

with $R / e<r<R$.

For this case, there are two solutions for the interior Bernoulli problem: one (called the "elliptic") solution which is the circle $C_{e}=\{x ;|x|=r\}$ and the other (called the "hyperbolic") solution which is the circle $C_{h}=\left\{x ;|x|=r_{h}\right\}$ where $r_{h}$ is the unique real number such that

$$
0<r_{h}<R / e, \quad \frac{1}{r_{h}\left(\log R-\log r_{h}\right)}=\lambda .
$$

Convergence to the elliptic or hyperbolic solution depends on the initial guess. In our test we are interested only in the elliptic solution. We take $R=0.9$ and $r=0.7$, so $\lambda=5.6844$. We take as an initial guess the circle centered at the origin of radius 0.4 . Table 2 shows the convergence test to the elliptic solution.

In the second test $\Upsilon_{0}$ is a L-shaped, the mesh grid is $202 \times 202, \lambda=14$ and the initial guess is the circle centered at $(-0.15,0.15)$ of radius 0.25 . We have obtained convergence after 48 iterations with $J\left(\Gamma^{48}\right)=0.0339$. Fig. 6 shows the position of $\Gamma^{k}$ at some iterations.

\section{Conclusion}

We have presented a new formulation of the Bernoulli problem: A shape optimization problem consisting in minimizing a constitutive error cost functional. We have numerically solved this optimization problem by a steepest descent algorithm using the gradient information combined with the level set method. Numerical tests illustrate the efficiency of the proposed approach.

\section{References}

[1] Acker A 1981, An extremal problem involving distributed resistance, SIAM J. Math. Anal. 12, $169-172$.

[2] Acker A, Meyer R 1995, A free boundary problem for the p-Laplacian: uniqueness, convexity and successive approximation of solutions, Electronic Journal of Differential Equations, 8 (1995), 1-20. MR 96c:35198. 

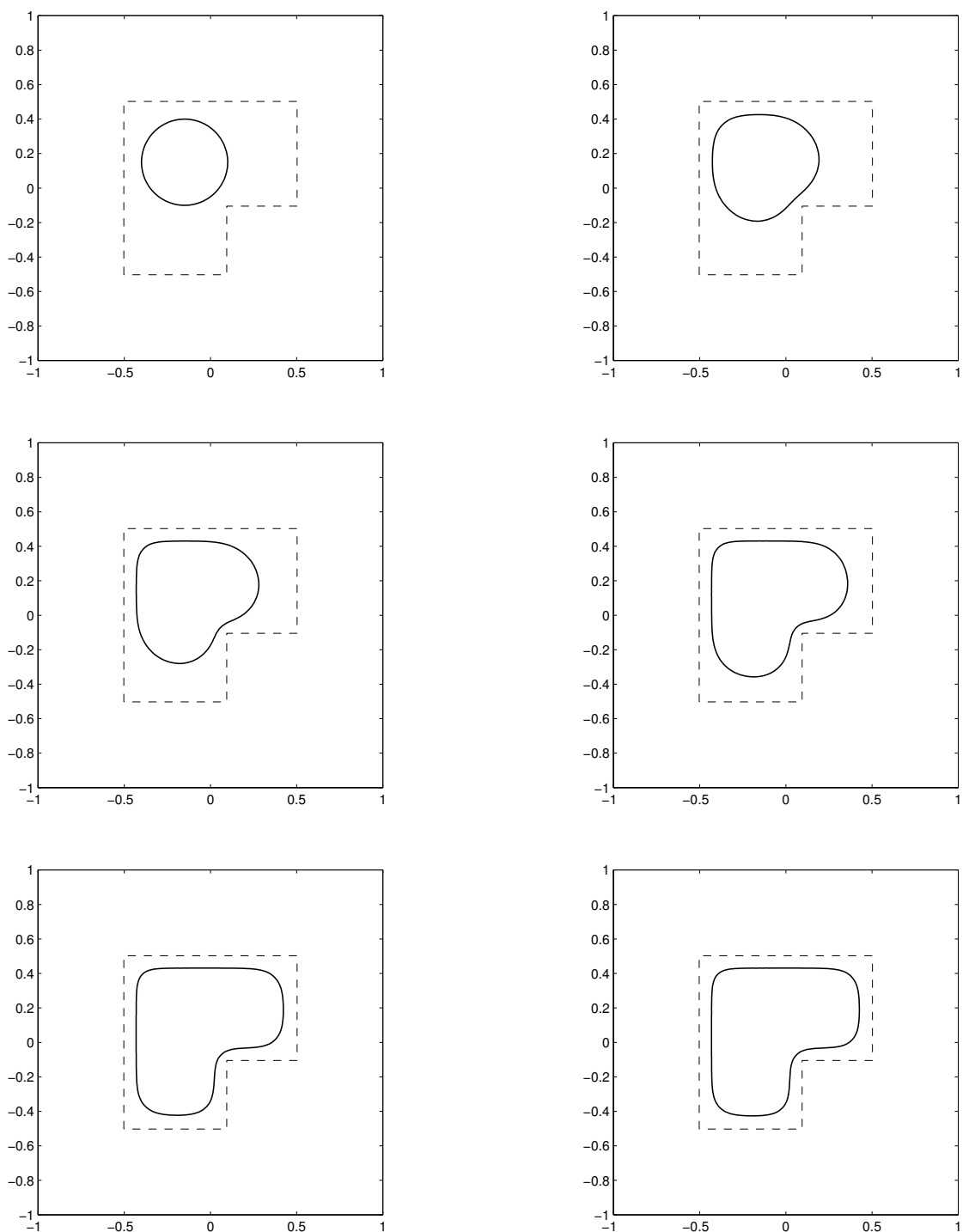

Figure 6. Convergence test for $\Upsilon_{0}$ an L-shaped (dashed line), position of $\Gamma^{k}$ (solid line) for $k=0,9,19,29,44,48$ (right to left and top to bottom).

[3] Alt A, Caffarelli LA 1981, Existence and regularity for a minimum problem with free boundary, J. Reine Angew Math. 325, 105-144.

[4] Adalsteinsson A and Sethian JA 1999, The fast construction of extension velocities in level set methods, J. Comp. Phys. 148, 2-22.

[5] Beurling A 1957, On free boundary problems for the Laplace equation, Seminars on analytic functions, 1, 248-263. Institute of Advance Studies Seminars, Princeton.

[6] Burger M 2001, A framework for the construction of level set methods for shape optimization and reconstruction, Inverse Problems 17, 1327-1356.

[7] Bouchon F, Clain S and Touzani R 2005, Numerical solution of the free boundary Bernoulli problem using a level set formulation, Comp. Meth. Appl. Mech. and Eng., 194, 3934-3948.

[8] Ben Abda A, Hassine M, Jaoua M and Masmoudi M 2009, Topological sensitivity analysis for the location of small cavities in Stokes flows, SIAM J. Cont. Opt., 48(5) 2871-2900.

[9] Chaabane S, Jaoua M 1999, Identification of Robin coefficients by means of boundary 
measurements, Inverse Problems, 15(6):1425.

[10] Delfour MC, Zolesio J-P 2001, Shapes and Geometries, SIAM.

[11] Fasano 1992, Some free boundary problems with industrial applications, in shape optimization and free boundaries (Edited by M. Delfour), Nato ASI Series, vol. 380. Kluwer.

[12] Friedman F 1984, Free boundary problem in fluid dynamics, Astérisque, Soc. Math. France, 118, $55-67$.

[13] Flucher M, Rumpf M 1997, Bernoulli's free-boundary problem, qualitative theory and numerical approximation, J. Reine Angew. Math. 486, 165-204.

[14] Haslinger J, Kozubek T, Kunish K and Peichl G 2003, Shape optimization and fictitious domain approach for solving free-boundary value problems of Bernoulli type, Comput. Optim. Appl., 26, 231-251.

[15] Henrot A, Shahgholian H 1997, Convexity of free boundaries with Bernoulli type boundary condition, Nonlinear Analysis, Theory, methods and Applications, 28, No. 5, 815-823.

[16] Ito K, Kunisch K and Peichl G 2006, Variational approach to shape derivative for a class of Bernoulli problem, J. Math. Anal. App., 314, 126-149.

[17] K. Eppler. 2000 Boundary integral representations of second derivatives in shape optimization, Discussiones Mathematicae (Differential Inclusions, Control and Optimization), 20:6378.

[18] K. Eppler. 2000 Optimal shape design for elliptic equations via BIE-methods J. of Applied Mathematics and Computer Science, 10:487516.

[19] K. Eppler and H. Harbrecht 2009 On a Kohn-Vogelius Like Formulation of Free Boundary Problems

[20] Kohn RV, McKenney A 1990, Numerical implementation of a variational method for electrical impedance tomography, Inverse Problems, 6(3):389.

[21] Lacey AA, Shillor M 1987, Electrochemical and electro-discharge machining with a threshold current, IMA J. Appl. Math., 39, 131-142.

[22] Ladeveze Pj, Leguillon D 1983, Error estimate procedure in the finite element method and applications, SIAM J. Num. Anal., 20(3):485-509.

[23] Murat F, Simon J 1976, Étude de problèmes d'optimal design, Lectures Notes in Computer Science, $41,54-62$.

[24] Osher S, Fedkiw R 2002, Level Set Methods and Dynamic Implicit Surfaces, Springer-Verlag, New York.

[25] Osher S, Sethian J, Fronts propagating with curvature dependent speed: Algorithms based on Hamilton-Jacobi formulations, J. Comp. Phys. 56, 1988, 12-49.

[26] Osher S, Santosa F 2001, Level set methods for optimization problems involving geometry and constraints, J. Comp. Phys., 171, 272-288.

[27] Sethian JA 1999, Level set methods and fast marching methods, Cambridge University Press.

[28] Sokolowski J, Zolésio J-P 1992, Introduction to Shape Optimization: Shape Sensitivity Analysis, Springer. 